LYCEN 9155

December 1991

\title{
Classical Trajectories for Two Ring-Shaped Potentials
}

\author{
MAURICE KIBLER AND GEORGES-HENRI LAMOT \\ Institut de Physique Nucléaire de Lyon, \\ IN2P3-CNRS et Université Claude Bernard, \\ F-69622 Villeurbanne Cedex, France
}

PAVEL WINTERNITZ

Centre de Recherches Mathématiques, Université de Montréal, CP 6128-A, Montréal, Québec, Canada H3C 3J7

This work [published in Int. J. Quantum Chem. 43, 625 (1992)] has been achieved in the framework of the France-Québec exchange programme (project no 200220 89). The kind hospitality extended to one of the authors (M. K.) at the Centre de Recherches Mathématiques de l'Université de Montréal on the occasion of several stays during the work on this project is gratefully acknowledged. The research of one of the authors (P. W.) is partially supported by research grants from NSERC of Canada and FCAR of Québec. Two of the authors (M. K. and P. W.) are indebted to Dr. N. W. Evans from the Queen Mary College (London) for sending them preprints on his work and to Professor Ya. A. Smorodinsky for an interesting discussion. 


\begin{abstract}
The present paper deals with the classical trajectories for two super-integrable systems : a system known in quantum chemistry as the Hartmann system and a system of potential use in quantum chemistry and nuclear physics. Both systems correspond to ringshaped potentials. They admit two maximally super-integrable systems as limiting cases, viz, the isotropic harmonic oscillator system and the Coulomb-Kepler system in three dimensions. The planarity of the trajectories is studied in a systematic way. In general, the trajectories are quasi-periodic rather than periodic. A constraint condition allows to pass from quasi-periodic motions to periodic ones. When written in a quantum mechanical context, this constraint condition leads to new accidental degeneracies for the two systems studied.
\end{abstract}




\section{Introduction}

Three-dimensional potentials that are singular along curves have received a great deal of attention in the recent years. In particular, the coulombic ring-shaped potential $V_{Q}$ (see Eq. (3.1)), revived in quantum chemistry by Hartmann and collaborators [1], and the oscillatory ring-shaped potential $U_{Q}$ (see Eq. 2.1)), systematically studied by Quesne [2], have been investigated from a quantum mechanical viewpoint by using various approaches (including Schrödinger, Feynman path integral, nonbijective canonical transformations, and Lie-like approaches). In this connection, we mention the works of Refs. [3-9] on the potential $V_{Q}$. Furthermore, the potential $U_{Q}$ and various extensions of $U_{Q}$ and $V_{Q}$ have been worked out by means of algebraic, path integral, and Lie-like techniques [10-14]. Finally, the so-called ABC and ABO systems (which are companions of the $V_{Q}$ and $U_{Q}$ systems concerning an Aharonov-Bohm plus a Coulomb potential and an Aharonov-Bohm plus an oscillator potential, respectively) have been the object of several investigations in a nonrelativistic formulation [15-18] as well as in a relativistic one [19].

The $U_{Q}$ and $V_{Q}$ systems have been little examined from a classical viewpoint $[7,20]$. It is the aim of this paper to study the classical motion for a (charged) particle embedded in the potential $U_{Q}$ or $V_{Q}$. More specifically, we shall investigate the planarity, the periodicity and the semi-classical quantization of the bounded motions for the $U_{Q}$ and $V_{Q}$ systems.

Among the potentials in $n=3$ dimensions, the potentials $U_{Q}$ and $V_{Q}$ have a special status in the sense that they correspond to super-integrable hamiltonian systems with $n+1=4$ integrals of motion. The latter two systems occupy a position intermediate between integrable hamiltonian systems (with $n=3$ constants) and maximally superintegrable hamiltonian systems (with $2 n-1=5$ constants). In addition, when the parameter $Q$ vanishes, the $U_{Q}$ and $V_{Q}$ systems become maximally super-integrable systems ; as a matter of fact, $U_{Q=0}$ corresponds to the oscillator system and $V_{Q=0}$ to the Coulomb-Kepler system. Let us recall that, in $n$ dimensions ( $n$ arbitrary), four maximally super-integrable

hamiltonian systems are known : the oscillator system, the Coulomb-Kepler system, the Calogero-Moser system, and a system introduced by Smorodinsky and co-workers [21,22] 
(see also Ref. [23]).

The $U_{Q}$ and $V_{Q}$ systems thus turn out to be two interesting laboratories among the super-integrable systems. As ring-shaped systems, they may play an important role in all situations where axial symmetry is relevant. For example, the $V_{Q}$ system is of interest for ring-shaped molecules like cyclic polyenes [1]. Further, the $U_{Q}$ system is of potential use in the study of (super-)deformed nuclei.

The plan of the present paper is as follows. For each of the potentials $U_{Q}$ and $V_{Q}$ we study in Sections 2 and 3, respectively, the constants of motion, the classical trajectories, their planarity, (quasi-)periodicity and quantization. The quasi-periodicity of the bounded motions is connected in Section 4 to the concept of "local" symmetry of a spectral problem as first introduced by Coulson's school in connection with the Kepler problem [24]. The planarity of the bounded motions is studied with the help of some new formulas, relegated to the appendix, for the torsion (and the curvature) of the trajectories of a given hamiltonian system.

\section{The Oscillatory Ring-Shaped Potential}

\subsection{Generalities}

We shall deal in this section with the potential (energy)

$$
U_{Q}=\frac{1}{2} \Omega^{2}\left(x_{1}^{2}+x_{2}^{2}+x_{3}^{2}\right)+\frac{1}{2} Q \frac{1}{x_{1}^{2}+x_{2}^{2}} \quad \Omega>0 \quad Q>0
$$

which clearly exhibits an $O(2)$ cylindrical symmetry. The limiting case $Q=0$ corresponds

to an isotropic harmonic oscillator (in $\mathbf{R}^{3}$ ) and will serve for testing the results to be obtained.

The potential $U_{Q}$ is a special case of the potential

$$
V_{3}=\alpha\left(x_{1}^{2}+x_{2}^{2}+x_{3}^{2}\right)+\beta \frac{1}{x_{3}^{2}}+h\left(\frac{x_{2}}{x_{1}}\right) \frac{1}{x_{1}^{2}+x_{2}^{2}}
$$


of Makarov et al. [22]. The potential $V_{3}$ allows the separation of variables for the HamiltonJacobi equation and, also, the Schrödinger equation in four systems of coordinates, viz, the spherical, circular cylindrical, prolate spheroidal and oblate spheroidal coordinates. According to Makarov et al. [22], there are four functionally independent integrals of motion for $V_{3}$ and, thus, for $U_{Q}$. More precisely, the four integrals for $U_{Q}$ can be constructed in terms of the relevant Hamilton function and the $A_{k}$ 's listed below $[2,20,22]$.

(i) Spherical coordinates :

$$
\begin{array}{cc}
x_{1}=r \sin \theta \cos \varphi \quad x_{2}=r \sin \theta \sin \varphi \quad x_{3}=r \cos \theta \\
A_{1}=\ell_{1}{ }^{2}+\ell_{2}{ }^{2}+\ell_{3}{ }^{2}+Q \frac{1}{\sin ^{2} \theta} \quad A_{3}=\ell_{3} .
\end{array}
$$

(ii) Circular cylindrical coordinates :

$$
\begin{gathered}
x_{1}=\rho \cos \varphi \quad x_{2}=\rho \sin \varphi \quad x_{3}=z \\
A_{2}=\frac{1}{2}\left({p_{3}}^{2}+\Omega^{2} x_{3}{ }^{2}\right) \quad A_{3}=\ell_{3} .
\end{gathered}
$$

(iii) Prolate spheroidal coordinates :

$$
x_{1}=a \sinh \eta \sin \alpha \cos \varphi \quad x_{2}=a \sinh \eta \sin \alpha \sin \varphi \quad x_{3}=a \cosh \eta \cos \alpha
$$

$$
A_{4}=\ell_{1}^{2}+\ell_{2}^{2}+\ell_{3}^{2}-a^{2}\left(p_{1}^{2}+{p_{2}}^{2}\right)-a^{4} \Omega^{2} \sinh ^{2} \eta \sin ^{2} \alpha+Q \frac{\sinh ^{2} \eta-\sin ^{2} \alpha}{\sinh ^{2} \eta \sin ^{2} \alpha} \quad A_{3}=\ell_{3}
$$

(iv) Oblate spheroidal coordinates :

$$
x_{1}=a \cosh \eta \sin \alpha \cos \varphi \quad x_{2}=a \cosh \eta \sin \alpha \sin \varphi \quad x_{3}=a \sinh \eta \cos \alpha
$$


$A_{5}=\ell_{1}^{2}+\ell_{2}^{2}+\ell_{3}^{2}+a^{2}\left(p_{1}^{2}+p_{2}^{2}\right)+a^{4} \Omega^{2} \cosh ^{2} \eta \sin ^{2} \alpha+Q \frac{\cosh ^{2} \eta+\sin ^{2} \alpha}{\cosh ^{2} \eta \sin ^{2} \alpha} \quad A_{3}=\ell_{3}$.

(It is important to note that, in order to adhere to standard notations, terms of type $\left(p_{k}\right)$ and $\left(-M_{k}\right.$ or $\left.L_{k}\right)$ in the notation of Makarov et al. [22] or Kibler and Winternitz [7] are replaced here by $\left(i p_{k}\right)$ and $\left(-i \ell_{k}\right)$, respectively. The variables $p_{k}$ and $\ell_{k}$ in the present paper stand for linear and angular momenta, respectively, in atomic units.)

Returning to Cartesian coordinates, we can write the first three integrals of motion as

$A_{1}=\ell_{1}^{2}+\ell_{2}^{2}+\ell_{3}^{2}+Q \frac{x_{1}^{2}+x_{2}^{2}+x_{3}^{2}}{x_{1}^{2}+x_{2}^{2}} \quad A_{2}=\frac{1}{2}\left(p_{3}^{2}+\Omega^{2} x_{3}^{2}\right) \quad A_{3}=x_{1} p_{2}-x_{2} p_{1}$.

The remaining two then satisfy

$$
A_{4}=A_{1}+2 a^{2}\left(A_{2}-H\right) \quad A_{5}=A_{1}-2 a^{2}\left(A_{2}-H\right)
$$

with

$$
H=\frac{1}{2}\left(p_{1}^{2}+p_{2}^{2}+p_{3}^{2}\right)+U_{Q}
$$

We see that $\left\{H, A_{1}, A_{2}, A_{3}\right\}$ form an integrity basis for all integrals of the considered Hamiltonian system.

A consequence of Eq. (2.5) is that any potential that allows the separation of variables in spherical and cylindrical coordinates will also allow the separation in prolate and oblate spheroidal coordinates. This holds both for the Hamilton-Jacobi and Schrödinger equations.

\subsection{Equipotential Surfaces}

The equipotentials corresponding to given values of $U_{Q}$, with $U_{Q} \geq \Omega \sqrt{Q}$, are described (in circular cylindrical coordinates) by

$$
z= \pm \frac{1}{\Omega} \frac{1}{\rho} \sqrt{-\Omega^{2} \rho^{4}+2 U_{Q} \rho^{2}-Q} \quad \rho=\sqrt{x_{1}^{2}+x_{2}^{2}} \quad z=x_{3}
$$


with the restrictions

$$
\rho_{<}^{2} \leq \rho^{2} \leq \rho_{>}^{2}
$$

where

$$
\rho_{<}{ }^{2}=\frac{U_{Q}-\sqrt{U_{Q}^{2}-\Omega^{2} Q}}{\Omega^{2}} \quad \rho_{>}{ }^{2}=\frac{U_{Q}+\sqrt{U_{Q}^{2}-\Omega^{2} Q}}{\Omega^{2}} .
$$

All the equipotentials corresponding to bounded values of $U_{Q}$ with $U_{Q}>\Omega \sqrt{Q}$ are bounded surfaces which collapse into simple lines (actually circles in the $z=0$ plane) for $U_{Q}=\Omega \sqrt{Q}$. Of course, each equipotential reduces to a simple sphere, of radius $\sqrt{2 U_{0}} / \Omega$, in the limiting situation $\left(Q=0, U_{0}>0\right)$.

\subsection{Trajectories}

We now derive the equations of motion for a particle with potential energy $U_{Q}$. We use here the cylindrical coordinates $q \equiv(\rho, \varphi, z)$ in which the Hamilton function $H$ for a particle of (reduced) mass $\mu=1$ in the potential $U_{Q}$ reads

$$
H \equiv H(p, q)=\frac{1}{2}\left(p_{\rho}{ }^{2}+\frac{1}{\rho^{2}} p_{\varphi}{ }^{2}+p_{z}{ }^{2}\right)+\frac{1}{2} \Omega^{2}\left(\rho^{2}+z^{2}\right)+\frac{1}{2} Q \frac{1}{\rho^{2}}
$$

where $p \equiv\left(p_{\rho}=\dot{\rho}, p_{\varphi}=\rho^{2} \dot{\varphi}, p_{z}=\dot{z}\right)$ are the relevant canonically conjugated momenta. The corresponding Hamilton-Jacobi equation can be written

$$
\frac{\partial S}{\partial t}+\frac{1}{2}\left[\left(\frac{\partial S}{\partial \rho}\right)^{2}+\frac{1}{\rho^{2}}\left(\frac{\partial S}{\partial \varphi}\right)^{2}+\left(\frac{\partial S}{\partial z}\right)^{2}\right]+\frac{1}{2} \Omega^{2}\left(\rho^{2}+z^{2}\right)+\frac{1}{2} Q \frac{1}{\rho^{2}}=0 .
$$

Obviously, $\varphi$ is a cyclic variable so that we put

$$
p_{\varphi}=\frac{\partial S}{\partial \varphi}=m \quad m \in \mathbf{R}
$$

and, since the system of the particle in $U_{Q}$ is conservative, we look for a solution of (2.11) in the form

$$
S=S_{1}(\rho)+m \varphi+S_{2}(z)-E t .
$$

This leads to the separated equations

$$
\frac{1}{2}\left(\frac{d S_{1}}{d \rho}\right)^{2}+\frac{1}{2} \Omega^{2} \rho^{2}+\frac{1}{2}\left(m^{2}+Q\right) \frac{1}{\rho^{2}}=K \quad \frac{1}{2}\left(\frac{d S_{2}}{d z}\right)^{2}+\frac{1}{2} \Omega^{2} z^{2}=-K+E
$$


which are coupled via a (positive) separation constant $K$. Equations (2.14) show that the only possible motions are bounded motions. They occur for

$$
E \geq K>0 \quad K^{2}-\Omega^{2} M^{2} \geq 0
$$

where

$$
M^{2}=m^{2}+Q
$$

Indeed, Eqs. (2.15) ensure that

$$
-z_{0} \leq z(t) \leq z_{0} \quad \rho_{1} \leq \rho(t) \leq \rho_{2}
$$

with

$$
z_{0}=\frac{\sqrt{2(E-K)}}{\Omega} \quad \rho_{1}=\frac{\sqrt{K-\sqrt{K^{2}-\Omega^{2} M^{2}}}}{\Omega} \quad \rho_{2}=\frac{\sqrt{K+\sqrt{K^{2}-\Omega^{2} M^{2}}}}{\Omega} .
$$

Direct integration of (2.14) yields

$$
\begin{gathered}
S_{1}(\rho)=\frac{1}{2} \Omega \sqrt{\left(\rho^{2}-\rho_{1}^{2}\right)\left(\rho_{2}^{2}-\rho^{2}\right)} \\
+\frac{1}{2} \frac{K}{\Omega} \sin ^{-1}\left[\frac{2 \rho^{2}-\left(\rho_{1}^{2}+\rho_{2}^{2}\right)}{\rho_{2}^{2}-\rho_{1}^{2}}\right]-\frac{1}{2}|M| \sin ^{-1}\left[\frac{\left(\rho_{1}^{2}+\rho_{2}^{2}\right) \rho^{2}-2 \rho_{1}^{2} \rho_{2}^{2}}{\left(\rho_{2}^{2}-\rho_{1}^{2}\right) \rho^{2}}\right] \\
S_{2}(z)=\frac{E-K}{\Omega}\left[\frac{z}{z_{0}} \sqrt{1-\left(\frac{z}{z_{0}}\right)^{2}}+\sin ^{-1}\left(\frac{z}{z_{0}}\right)\right] .
\end{gathered}
$$

Equation $(2.19 a)$ is valid for $K^{2}-\Omega^{2} M^{2}>0$; in the special case $K^{2}-\Omega^{2} M^{2}=0$, we have

$$
\rho_{1}=\rho_{2} \equiv \rho_{0}=\frac{\sqrt{K}}{\Omega}=\frac{|M|}{\sqrt{K}}=\sqrt{\frac{|M|}{\Omega}} \Rightarrow S_{1}(\rho)=\text { constant }
$$

and the motion is constrained to $\rho=\rho_{0}$.

The action $S$ follows from (2.13) and (2.19). Therefore, the equations of motion

$$
\frac{\partial S}{\partial E}=\beta_{1} \quad \frac{\partial S}{\partial K}=\beta_{2} \quad \frac{\partial S}{\partial m}=\beta_{3}
$$

can be derived in a straightforward way. In fact, in view of the relations

$$
\beta_{1}=\frac{\partial S_{2}}{\partial E}-t \quad \beta_{2}=\frac{\partial S_{1}}{\partial K}+\frac{\partial S_{2}}{\partial K}=\frac{\partial S_{1}}{\partial K}-\frac{\partial S_{2}}{\partial E} \quad \beta_{3}=\frac{\partial S_{1}}{\partial m}+\varphi
$$


we choose $t_{0}^{\prime} \equiv-\beta_{1}, t_{0} \equiv-\left(\beta_{1}+\beta_{2}\right)$ and $\varphi_{0} \equiv \beta_{3}$ for constants of motion. Thus, we obtain (for $K^{2}-\Omega^{2} M^{2}>0$ )

$$
\begin{gathered}
t-t_{0}^{\prime}=\frac{1}{\Omega} \sin ^{-1}\left(\frac{z}{z_{0}}\right) \\
t-t_{0}=\frac{1}{2 \Omega} \sin ^{-1}\left[\frac{2 \rho^{2}-\left(\rho_{1}^{2}+\rho_{2}^{2}\right)}{\rho_{2}^{2}-\rho_{1}^{2}}\right] \\
\varphi-\varphi_{0}=\frac{1}{2} \frac{m}{|M|} \sin ^{-1}\left[\frac{\left(\rho_{1}^{2}+\rho_{2}^{2}\right) \rho^{2}-2 \rho_{1}^{2} \rho_{2}^{2}}{\left(\rho_{2}^{2}-\rho_{1}^{2}\right) \rho^{2}}\right]
\end{gathered}
$$

from which we can extract the coordinates $\rho(t), \varphi(t)$ and $z(t)$. We finally arrive at

$$
\begin{gathered}
\rho(t)=\frac{1}{\sqrt{2}} \sqrt{\rho_{1}^{2}+\rho_{2}^{2}+\left(\rho_{2}^{2}-\rho_{1}^{2}\right) \sin \left[2 \Omega\left(t-t_{0}\right)\right]} \\
\varphi(t)=\varphi_{0}+\frac{1}{2} \frac{m}{|M|} \sin ^{-1}\left\{\frac{\rho_{2}^{2}-\rho_{1}^{2}+\left(\rho_{1}^{2}+\rho_{2}^{2}\right) \sin \left[2 \Omega\left(t-t_{0}\right)\right]}{\rho_{1}^{2}+\rho_{2}^{2}+\left(\rho_{2}^{2}-\rho_{1}^{2}\right) \sin \left[2 \Omega\left(t-t_{0}\right)\right]}\right\} \\
z(t)=z_{0} \sin \left[\Omega\left(t-t_{0}^{\prime}\right)\right]
\end{gathered}
$$

in agreement with a previous derivation based on functionally independent constants of motion [20]. Equations (2.24a) and (2.24b) are valid for $K^{2}-\Omega^{2} M^{2}>0$ and also for $K^{2}-\Omega^{2} M^{2}=0$ under the condition $\rho_{1}=\rho_{2}=\rho_{0}$. Note that in $(2.24 b)$, the parameter $m /|M|$ (which is \pm 1 in the limiting case $Q=0$ ) is given by

$$
\frac{m^{2}}{M^{2}}=1-\frac{Q}{\Omega^{2}} \frac{1}{\rho_{1}^{2} \rho_{2}^{2}}
$$

so that the variables $\rho, \varphi$ and $z$ depend on the six constants $\rho_{1}, \rho_{2}, \varphi_{0}, z_{0}, t_{0}$ and $t_{0}^{\prime}$.

At this stage, it is worth mentioning that $(2.24 b)$ is equivalent to

$$
\varphi(t)=\varphi_{0}-\frac{1}{2} \frac{m}{|M|} \alpha+\frac{m}{|M|} \tan ^{-1}\left\{\frac{\left(\rho_{1}^{2}+\rho_{2}^{2}\right) \tan \left[\Omega\left(t-t_{0}\right)\right]+\rho_{2}^{2}-\rho_{1}^{2}}{2 \rho_{1} \rho_{2}}\right\}
$$

with

$$
\sin \alpha=\frac{\rho_{2}^{2}-\rho_{1}^{2}}{\rho_{1}^{2}+\rho_{2}^{2}} \quad \cos \alpha=\frac{2 \rho_{1} \rho_{2}}{\rho_{1}^{2}+\rho_{2}^{2}} .
$$

Indeed, Eq. (2.26) naturally arises when solving the Newton equations for the dynamical system under consideration. 
As a résumé, all the trajectories are bounded. The most general motion corresponds to

$$
\Omega>0 \quad Q>0 \quad E>K>\Omega|M|>0 .
$$

It follows from (2.17) that the classical trajectories lay between two cylinders of height $2 z_{0}$ and radii $\rho_{1}$ and $\rho_{2}$. The real constant $m$ is the $z$-component $\ell_{3}$ of the angular momentum for the system being studied ; we thus recover that $M^{2}=A_{3}{ }^{2}+Q$ is a constant of motion (cf. Ref. [22]). Furthemore, the positive constant $K$ is another constant of motion, namely, $K=E-A_{2}$. In terms of $\rho_{1}, \rho_{2}$ and $z_{0}$, the three constants of motion $m, K$ and $E$ are given by

$$
m^{2}=\Omega^{2}{\rho_{1}}^{2} \rho_{2}{ }^{2}-Q \quad K=\frac{1}{2} \Omega^{2}\left(\rho_{1}{ }^{2}+\rho_{2}{ }^{2}\right) \quad E=\frac{1}{2} \Omega^{2}\left(\rho_{1}{ }^{2}+\rho_{2}{ }^{2}+z_{0}{ }^{2}\right) .
$$

Finally, it is to be emphasized that the trajectories are neither periodic nor planar in general.

\subsection{Planarity of Trajectories}

The question of planarity of (bounded and nonbounded) motions can be tackled by looking at the torsion $\tau$ of the trajectories. A planar motion corresponds to $\tau=0$ along the trajectory. For the $U_{Q}$ system, by using the formula for $\tau=-\mathrm{NUM} / \mathrm{DEN}$ derived in the appendix, we get

$$
\begin{aligned}
\rho^{8} \times \mathrm{NUM} & =Q m\left[\left(Q-\Omega^{2} \rho^{4}\right) \dot{z}+4 \Omega^{2} \rho^{2}(x \dot{x}+y \dot{y}) z\right] \\
\rho^{8} \times \mathrm{DEN} & =\left(Q-\Omega^{2} \rho^{4}\right)^{2}\left(m^{2}+\rho^{2} \dot{z}^{2}\right) \\
& +2 \Omega^{2} \rho^{4}\left(Q-\Omega^{2} \rho^{4}\right)(x \dot{x}+y \dot{y}) z \dot{z}+\left(\Omega^{2} \rho^{4}\right)^{2}\left(\dot{x}^{2}+\dot{y}^{2}\right) z^{2} .
\end{aligned}
$$

The condition $\tau=0$ can be seen to be equivalent to

$$
Q m\left[\left(Q-\Omega^{2} \rho^{4}\right) \dot{z}+4 \Omega^{2} \rho^{3} \dot{\rho} z\right]=Q m\left[\left(Q-\Omega^{2} \rho^{4}\right) \frac{d}{d t}(z)-z \frac{d}{d t}\left(Q-\Omega^{2} \rho^{4}\right)\right]=0
$$

with

$$
\rho \dot{\rho}=x \dot{x}+y \dot{y}= \pm \sqrt{\left[2 E-\dot{z}^{2}-\Omega^{2}\left(\rho^{2}+z^{2}\right)\right] \rho^{2}-M^{2}} .
$$

Solutions of Eq. (2.31) are : 
(i) $Q=0$. In this case the studied system reduces to the harmonic oscillator for which of course all trajectories are planar (ellipses with the attractive "sun" in the center).

(ii) $z=\dot{z}=0$. We then have $z_{0}=0$ in (2.24c) and hence $E=K$, see (2.29).

(iii) $m=0$. The motion is restricted to the plane $\varphi=\varphi_{0}$, see $(2.24 \mathrm{~b})$ and $(2.26)$, and the limiting radii satisfy $\rho_{1} \rho_{2}=\sqrt{Q} / \Omega$, see $(2.29)$.

(iv) $\rho^{4}=Q / \Omega^{2}$. Indeed, the generic solution of (2.31) is $Q-\Omega^{2} \rho^{4}=c_{0} z$ with $c_{0}=$ constant. However, since $z$ and $\rho$ have different periods, see (2.24), the latter solution can only hold for $\rho=\rho_{1}=\rho_{2}=\left(Q / \Omega^{2}\right)^{1 / 4}$ which implies $m=0$, see (2.29), and hence either $z_{0}=0$ (no motion) or $c_{0}=0$.

To sum up, excluding the well known harmonic oscillator corresponding to the limiting case $Q=0$, we find that the trajectories are planar only if we have $m=0$ or $E=K$.

\subsection{Periodicity and Quasi-Periodicity}

From Eq. (2.23), we easily see that the projections onto the $x y$ plane of the trajectories are described by

$$
\rho=\sqrt{2} \frac{\rho_{1} \rho_{2}}{\sqrt{\rho_{1}^{2}+\rho_{2}^{2}-\left(\rho_{2}^{2}-\rho_{1}^{2}\right) \sin \left[2 \frac{|M|}{m}\left(\varphi-\varphi_{0}\right)\right]}}
$$

and, thus, these projections are not closed in general. Equation (2.33) shows that the projection onto the $x y$ plane of a given trajectory is closed if the condition

$$
\frac{|M|}{m}=\frac{k_{1}}{k_{2}} \quad k_{1} \in \mathbf{Z} \quad k_{2} \in \mathbf{Z}
$$

(with $k_{1}{ }^{2}>{k_{2}}^{2}$ ) is fulfilled. The situation $k_{1}{ }^{2}=k_{2}{ }^{2}=1$ corresponds to the limiting case $Q=0$ for which we know that all trajectories (actually ellipses) are planar and periodic. In the situation where $(2.34)$ is satisfied, then not only the projection, onto the $x y$ plane, of the trajectory is periodic, but the trajectory itself is periodic too. The period of the motion is then

$$
T=k_{1} T_{O} \quad T_{O}=\frac{2 \pi}{\Omega}
$$


where $T_{O}$ is the oscillator period corresponding to the limiting case $Q=0$. (See also Ref. [20] for an alternative derivation of (2.35).)

In the general case $Q>0$, when the condition (2.34) is satisfied, we have

$$
m^{2}=Q \frac{k_{2}{ }^{2}}{{k_{1}}^{2}-{k_{2}}^{2}} \quad \rho_{1}^{2} \rho_{2}{ }^{2}=\frac{Q}{\Omega^{2}} \frac{k_{1}{ }^{2}}{{k_{1}}^{2}-k_{2}{ }^{2}}
$$

for the periodic motions. The requirement that the trajectories be periodic thus leads to a "quantization condition" for the component $\ell_{3}$ of the angular momentum and the mean geometric radius $\sqrt{\rho_{1} \rho_{2}}$. Motions for which $(2.34)$ is not satisfied are quasi-periodic motions rather than periodic ones.

The potential energy

$$
\overline{U_{Q}}=\frac{1}{T_{O}} \int_{0}^{T_{O}}\left[\frac{1}{2} \Omega^{2}\left(\rho^{2}+z^{2}\right)+\frac{1}{2} Q \frac{1}{\rho^{2}}\right] d t
$$

averaged over a duration $T_{O}$ (a common period for $\rho$ and $z$ ) can be easily calculated from $(2.24 a)$ and $(2.24 c)$. We get

$$
\overline{U_{Q}}=\frac{1}{4} \Omega^{2}\left(\rho_{1}{ }^{2}+\rho_{2}{ }^{2}+z_{0}{ }^{2}\right)+\frac{1}{2} Q \frac{1}{\rho_{1} \rho_{2}} \Rightarrow \overline{U_{Q}}=\frac{1}{2} E+\frac{1}{2} Q \frac{1}{\rho_{1} \rho_{2}}
$$

for periodic and quasi-periodic motions. Thus, the virial theorem $(\bar{T}=\bar{V}=E / 2)$ for the three-dimensional isotropic harmonic oscillator applies to the $U_{Q}$ system only when $Q=0$.

\subsection{Particular Cases}

The case $Q=0$. As a general check of the correctness of $(2.24 a),(2.24 b)$ (or (2.26)) and $(2.24 c)$, we can verify, by calculating $x=\rho \cos \varphi$ and $y=\rho \sin \varphi$, that the limiting case $Q=0$ effectively corresponds to a three-dimensional isotropic harmonic oscillator with angular frequency $\Omega$. As a further check, it can be verified that for $Q=0$ the components of the angular momentum for the considered particle are

$$
\begin{aligned}
& \ell_{2}=-\frac{\Omega}{\sqrt{2}} z_{0} \sqrt{\rho_{1}^{2}+\rho_{2}^{2}}\left\{\cos \left(\varphi_{0}+\frac{\alpha}{2}\right) \cos \left[\Omega\left(t_{0}^{\prime}-t_{0}\right)\right]-\sin \left(\varphi_{0}-\frac{\alpha}{2}\right) \sin \left[\Omega\left(t_{0}^{\prime}-t_{0}\right)\right]\right\} \\
& \ell_{3}=m \\
& \ell_{1}=+\frac{\Omega}{\sqrt{2}} z_{0} \sqrt{\rho_{1}^{2}+\rho_{2}^{2}}\left\{\sin \left(\varphi_{0}+\frac{\alpha}{2}\right) \cos \left[\Omega\left(t_{0}^{\prime}-t_{0}\right)\right]+\cos \left(\varphi_{0}-\frac{\alpha}{2}\right) \sin \left[\Omega\left(t_{0}^{\prime}-t_{0}\right)\right]\right\}
\end{aligned}
$$


indicating that the elliptic trajectory corresponding to $\rho_{1}, \rho_{2}, \varphi_{0}, z_{0}, t_{0}$ and $t_{0}^{\prime}$ is in the plane perpendicular to the constant vector $\left(\ell_{1}, \ell_{2}, \ell_{3}\right)$.

The case $K=E$. Equations (2.18) and (2.24c) show that the trajectories are in the $x y$ plane when $E=K$, a situation that corresponds to case (ii) of section 2.4. They are described by (2.33) and thus are not closed in general. However, the planar trajectories corresponding to $E=K$ become closed if the condition (2.34) is satisfied.

The case $m=0$. Case (iii) of section 2.4 indicates that the trajectories are planar for $m=0$ and, from Eqs. (2.24), we see that they are in the $\rho z$ plane corresponding to $\varphi(t)=\varphi_{0}$. All the trajectories $(\rho(t), z(t))$ are periodic, of period $T_{O}$.

The case $K=\Omega|M|$. The two cylinders of radii $\rho_{1}$ and $\rho_{2}$ collapse into a single one, of radius $\rho_{0}$, when $K=\Omega|M|$; therefore, the trajectories (neither closed nor planar in general) are on a cylinder of height $2 z_{0}$ and radius $\rho_{0}$ (see $(2.17),(2.18)$ and $(2.20)$ ). Then, Eqs. (2.24) lead to the Cartesian coordinates

$$
x(t)=\rho_{0} \cos \left(\Omega \frac{m}{|M|} t+\varphi_{0}^{\prime}\right) \quad y(t)=\rho_{0} \sin \left(\Omega \frac{m}{|M|} t+\varphi_{0}^{\prime}\right) \quad z(t)=z_{0} \sin \left[\Omega\left(t-t_{0}^{\prime}\right)\right]
$$

where $\varphi_{0}^{\prime}$ is some new constant. The motion thus results from the combination of a circular motion of angular frequency

$$
\omega=\Omega \frac{m}{|M|}=\frac{m}{\rho_{0}^{2}}
$$

in the $x y$ plane and of an oscillatory motion of angular frequency $\Omega$ in the $z$ direction. (Without loss of generality, we can take $m>0$; the case $m=0$ is trivial.) In the limiting case $Q=0$, the trajectory reduces to an ellipse, the projection of which on the $x y$ plane is a circle of radius $\rho_{0}$.

In the case $K=\Omega|M|$ and $Q>0$, the trajectories are not closed in general. However, if we further assume that the condition (2.34) is satisfied, the trajectories become closed and of period $T=k_{1} T_{O}$. They are given by (2.40) and (2.41) with

$$
\frac{\Omega}{\omega}=\frac{k_{1}}{k_{2}} \quad \rho_{0}=\sqrt[4]{\frac{Q}{\Omega^{2}}} \sqrt[4]{\frac{k_{1}^{2}}{k_{1}^{2}-k_{2}^{2}}}
$$


with $k_{1}>k_{2}, k_{1} \in \mathbf{N}-\{0,1\}$ and $k_{2} \in \mathbf{N}-\{0\}$.

\subsection{Semi-Classical Quantization}

We now use the Bohr-Sommerfeld-Kramers quantization conditions to derive, in a semi-classical way, the quantum mechanical spectrum for the $U_{Q}$ system. Here, these conditions read

$$
\oint p_{\rho} d \rho=\left(n_{\rho}+\epsilon_{\rho}\right) 2 \pi \quad n_{\rho} \in \mathbf{N} \quad \oint p_{z} d z=\left(n_{z}+\epsilon_{z}\right) 2 \pi \quad n_{z} \in \mathbf{N}
$$

where $\epsilon_{\rho}$ and $\epsilon_{z}$ stand for (rational) numbers to reconcile the old and new theories of quanta. (In the case of a one-dimensional harmonic oscillator, we know that $\epsilon_{z}=1 / 2$.) In other words, we have

$$
2\left[S_{1}\left(\rho_{2}\right)-S_{1}\left(\rho_{1}\right)\right]=\left(n_{\rho}+\epsilon_{\rho}\right) 2 \pi \quad 2\left[S_{2}\left(z_{0}\right)-S_{2}\left(-z_{0}\right)\right]=\left(n_{z}+\epsilon_{z}\right) 2 \pi
$$

and, from (2.19), we obtain

$$
\frac{1}{2} \frac{K}{\Omega}-\frac{1}{2}|M|=n_{\rho}+\epsilon_{\rho} \quad \frac{E-K}{\Omega}=n_{z}+\epsilon_{z} .
$$

Consequently, the eigenvalue $E$ is given by

$$
E=\left(|M|+2 n_{\rho}+n_{z}+\frac{3}{2}\right) \Omega \quad|M|=\sqrt{m^{2}+Q} \quad m \in \mathbf{Z} \quad n_{\rho} \in \mathbf{N} \quad n_{z} \in \mathbf{N}
$$

in agreement with the result by Quesne [2]. In fact, Eq. (2.46) demands that $\epsilon_{z}+2 \epsilon_{\rho}=3 / 2$, a relation which is reminiscent of the three-dimensional isotropic harmonic oscillator.

\section{The Coulombic Ring-Shaped Potential}

\subsection{Generalities}

The coulombic ring-shaped, or Hartmann, potential (energy) is

$$
V_{Q}=-Z \frac{1}{\sqrt{x_{1}^{2}+x_{2}^{2}+x_{3}^{2}}}+\frac{1}{2} Q \frac{1}{x_{1}^{2}+x_{2}^{2}} \quad Z>0 \quad Q>0
$$


where $Z=\eta \sigma^{2}$ and $Q=q \eta^{2} \sigma^{2}$ in the notation of Hartmann [1] and of Kibler and Négadi [3]. Such an $O(2)$ invariant potential reduces to an attractive Coulomb potential (in $\mathbf{R}^{3}$ ) in the limiting case $Q=0$ and this will prove useful for checking purposes.

Clearly, $V_{Q}$ is a special case of the potential (in spherical coordinates)

$$
V_{4}=\alpha \frac{1}{r}+\beta \frac{\cos \theta}{r^{2} \sin ^{2} \theta}+h(\tan \varphi) \frac{1}{r^{2} \sin ^{2} \theta}
$$

introduced by Makarov et al. [22]. Therefore, the Schrödinger equation and, thus, the Hamilton-Jacobi equation for $V_{Q}$ are separable in spherical and parabolic rotational coordinates. In this respect, let us simply recall that there are four functionally independent integrals of motion for $V_{Q}$ which can be obtained from the relevant Hamilton function and the $B_{k}$ 's given below $[7,20,22]$.

(i) Spherical coordinates :

$$
\begin{array}{cc}
x_{1}=r \sin \theta \cos \varphi \quad x_{2}=r \sin \theta \sin \varphi \quad x_{3}=r \cos \theta \\
B_{1}=\ell_{1}{ }^{2}+\ell_{2}{ }^{2}+\ell_{3}{ }^{2}+Q \frac{1}{\sin ^{2} \theta} \quad B_{2}=\ell_{3} .
\end{array}
$$

(ii) Parabolic rotational coordinates:

$$
\begin{gathered}
x_{1}=\sqrt{a b} \cos \varphi \quad x_{2}=\sqrt{a b} \sin \varphi \quad x_{3}=\frac{1}{2}(a-b) \\
B_{3}=\frac{1}{2}\left(\ell_{1} p_{2}+p_{2} \ell_{1}-\ell_{2} p_{1}-p_{1} \ell_{2}+2 Z \frac{a-b}{a+b}-Q \frac{a-b}{a b}\right) \quad B_{2}=\ell_{3} .
\end{gathered}
$$

(The constant $B_{3}$ is given in a quantum mechanical form. The factor $+2[\ldots]$ in the corresponding expression of Ref. [22] for $B_{3}$ should be changed into -2[...].)

\subsection{Equipotential Surfaces}


The equipotentials are formally given (in circular cylindrical coordinates) by

$$
z= \pm \frac{\rho}{Q-2 V_{Q} \rho^{2}} \sqrt{4 Z^{2} \rho^{2}-\left(Q-2 V_{Q} \rho^{2}\right)^{2}} \quad \rho=\sqrt{x_{1}^{2}+x_{2}^{2}} \quad z=x_{3} .
$$

In contradistinction with $U_{Q}$, three cases must be considered for the potential $V_{Q}$.

(i) The case $-(1 / 2)\left(Z^{2} / Q\right)<V_{Q}<0$ : The equipotentials are given by (3.5) with the restrictions

$$
\rho_{<} \leq \rho \leq \rho_{>} \quad \rho_{<}=\frac{Z-\sqrt{Z^{2}+2 Q V_{Q}}}{-2 V_{Q}} \quad \rho_{>}=\frac{Z+\sqrt{Z^{2}+2 Q V_{Q}}}{-2 V_{Q}}
$$

and each equipotential is a bounded surface which reduces to a circle in the $z=0$ plane for $V_{Q}=-(1 / 2)\left(Z^{2} / Q\right)$. In the limiting case $Q=0$, each equipotential for $V_{0}<0$ becomes a sphere of radius $-Z / V_{0}$.

(ii) The case $V_{Q}=0$ : The equipotentials are obtained from

$$
z= \pm \frac{1}{Q} \rho \sqrt{4 Z^{2} \rho^{2}-Q^{2}}
$$

with the restriction

$$
\rho \geq \frac{1}{2} \frac{Q}{Z}
$$

and thus the equipotentials are not bounded surfaces in this case. (The limiting case $Q=0$ yields of course a sphere of infinite radius.)

(iii) The case $V_{Q}>0$ : The equipotentials are given by (3.5) with the restriction

$$
\rho_{<} \leq \rho<\rho_{>} \quad \rho_{<}=\frac{-Z+\sqrt{Z^{2}+2 Q V_{Q}}}{2 V_{Q}} \quad \rho_{>}=\frac{1}{\sqrt{2}} \sqrt{\frac{Q}{V_{Q}}}
$$

and here again all the equipotentials are bounded surfaces.

\subsection{Trajectories}

3.3.1. Preliminaries. We shall solve the Hamilton-Jacobi equation for $V_{Q}$ in spherical coordinates. The general pattern to be followed for $V_{Q}$ resembles the one for $U_{Q}$ so that we shall mention only the basic steps. 
The Hamilton-Jacobi equation for a particle of reduced mass $\mu=1$ in $V_{Q}$ can be written as

$$
\frac{\partial S}{\partial t}+\frac{1}{2}\left[\left(\frac{\partial S}{\partial r}\right)^{2}+\frac{1}{r^{2}}\left(\frac{\partial S}{\partial \theta}\right)^{2}+\frac{1}{r^{2} \sin ^{2} \theta}\left(\frac{\partial S}{\partial \varphi}\right)^{2}\right]-Z \frac{1}{r}+\frac{1}{2} Q \frac{1}{r^{2} \sin ^{2} \theta}=0
$$

and a solution of the type

$$
S=S_{1}(r)+S_{2}(\theta)+m \varphi-E t
$$

is easily found by solving the two equations

$$
r^{2}\left(\frac{d S_{1}}{d r}\right)^{2}-2 E r^{2}-2 Z r=-K \quad\left(\frac{d S_{2}}{d \theta}\right)^{2}+\left(m^{2}+Q\right) \frac{1}{\sin ^{2} \theta}=K
$$

where $K$ is a (positive) separation constant. Equations (3.12) show that two kinds of motions may occur here : nonbounded motions for $E \geq 0$ and bounded motions for

$$
-\frac{1}{2} \frac{Z^{2}}{K} \leq E<0
$$

We shall restrict ourselves to the bounded motions and to the separatrix which corresponds to $E=0$. As in the case for $U_{Q}$, the equations of motion are given by (2.21) and we shall use the constant $M$ defined via (2.16).

3.3.2. Bounded motions. They are confined in a region delimited by

$$
\begin{gathered}
r_{1} \leq r(t) \leq r_{2} \quad r_{1}=\frac{Z-\sqrt{Z^{2}+2 E K}}{-2 E} \quad r_{2}=\frac{Z+\sqrt{Z^{2}+2 E K}}{-2 E} \\
\theta_{0} \leq \theta(t) \leq \pi-\theta_{0} \quad \sin \theta_{0}=\frac{|M|}{\sqrt{K}} .
\end{gathered}
$$

By assuming that $-(1 / 2)\left(Z^{2} / K\right)<E<0$, Eqs. (3.12) admit the solutions

$$
\begin{aligned}
S_{1}(r) & =(-2 E)^{1 / 2} \sqrt{\left(r-r_{1}\right)\left(r_{2}-r\right)} \\
& +(-2 E)^{-1 / 2} Z \sin ^{-1}\left[\frac{2 r-\left(r_{1}+r_{2}\right)}{r_{2}-r_{1}}\right]-\sqrt{K} \sin ^{-1}\left[\frac{\left(r_{1}+r_{2}\right) r-2 r_{1} r_{2}}{\left(r_{2}-r_{1}\right) r}\right]
\end{aligned}
$$




$$
\begin{aligned}
S_{2}(\theta)= & \frac{|M|}{2}\left\{\sin ^{-1}\left[\frac{1}{\cos \theta_{0}}\left(-1+\frac{\sin ^{2} \theta_{0}}{1-\cos \theta}\right)\right]-\sin ^{-1}\left[\frac{1}{\cos \theta_{0}}\left(-1+\frac{\sin ^{2} \theta_{0}}{1+\cos \theta}\right)\right]\right\} \\
& -\sqrt{K} \sin ^{-1}\left(\frac{\cos \theta}{\cos \theta_{0}}\right) .
\end{aligned}
$$

Putting $t_{0} \equiv-\beta_{1}, \beta_{0} \equiv-2 \sqrt{K} \beta_{2}$ and $\varphi_{0} \equiv \beta_{3}$, we get from $(2.21)$

$$
\begin{gathered}
t-t_{0}=-(-2 E)^{-1 / 2} \sqrt{\left(r-r_{1}\right)\left(r_{2}-r\right)}+Z(-2 E)^{-3 / 2} \sin ^{-1}\left[\frac{2 r-\left(r_{1}+r_{2}\right)}{r_{2}-r_{1}}\right] \quad(3.16 a) \\
\beta_{0}=\sin ^{-1}\left(\frac{\cos \theta}{\cos \theta_{0}}\right)+\sin ^{-1}\left[\frac{\left(r_{1}+r_{2}\right) r-2 r_{1} r_{2}}{\left.r_{2}-r_{1}\right) r}\right] \\
\varphi-\varphi_{0}=\frac{1}{2} \frac{m}{|M|}\left\{\sin ^{-1}\left[\frac{1}{\cos \theta_{0}}\left(-1+\frac{\sin ^{2} \theta_{0}}{1+\cos \theta}\right)\right]-\sin ^{-1}\left[\frac{1}{\cos \theta_{0}}\left(-1+\frac{\sin ^{2} \theta_{0}}{1-\cos \theta}\right)\right]\right\} .
\end{gathered}
$$

Equation (3.16b) is amenable to the form

$$
r \cos \theta=\frac{\cos \theta_{0}}{r_{2}-r_{1}}\left\{\left[2 r_{1} r_{2}-\left(r_{1}+r_{2}\right) r\right] \cos \beta_{0}+2 \sqrt{r_{1} r_{2}} \sqrt{\left(r-r_{1}\right)\left(r_{2}-r\right)} \sin \beta_{0}\right\}
$$

and, therefore, Eqs. (3.16) are in accordance with the ones derived by Kibler and Winternitz [20] from functionally independent constants of motion. Equations (3.16) are valid for $-(1 / 2)\left(Z^{2} / K\right)<E<0$. The case $E=-(1 / 2)\left(Z^{2} / K\right)<0$ deserves a particular study (see section 3.6). In Eq. (3.16c), note that the parameter $m /|M|$ (which is \pm 1 in the limiting case $Q=0$ ) can be obtained from

$$
\frac{m^{2}}{M^{2}}=1-\frac{1}{2} \frac{Q}{Z} \frac{r_{1}+r_{2}}{r_{1} r_{2}} \frac{1}{\sin ^{2} \theta_{0}}
$$

so that the variables $r, \theta$ and $\varphi$ depend on the six constants $r_{1}, r_{2}, \theta_{0}, \varphi_{0}, t_{0}$ and $\beta_{0}$.

To sum up, the most general bounded motion corresponds to

$$
Z>0 \quad Q>0 \quad-\frac{1}{2} \frac{Z^{2}}{K}<E<0
$$

and all finite trajectories take place between two spheres of radii $r_{1}$ and $r_{2}$ (see (3.14)). The interpretation of the constant $m$ (or $M$ ) is similar to the one for $U_{Q}$ : we have $M^{2}=B_{2}{ }^{2}+Q$. Furthermore, it can be verified that $K=B_{1}$. In terms of $r_{1}, r_{2}$ and $\theta_{0}$, the three constants of motion $m, K$ and $E$ can be deduced from

$$
m^{2}=2 Z \frac{r_{1} r_{2}}{r_{1}+r_{2}} \sin ^{2} \theta_{0}-Q \quad K=2 Z \frac{r_{1} r_{2}}{r_{1}+r_{2}} \quad E=-Z \frac{1}{r_{1}+r_{2}} .
$$


Here again, the finite trajectories are neither periodic nor planar in general.

3.3.3. The separatrix. By taking $E=0$, Eqs. (3.12) lead to

$$
r(t) \geq r_{\text {min }} \quad r_{\text {min }}=\frac{1}{2} \frac{K}{Z} \quad \theta_{0} \leq \theta(t) \leq \pi-\theta_{0} \quad \sin \theta_{0}=\frac{|M|}{\sqrt{K}}
$$

so that the motions are nonbounded. From (3.11) and (3.12), it appears that the coordinate $\varphi(t)$ is still given by $(3.16 c)$ when $E=0$. In addition, the coordinate $r(t)$ can be determined from the cubic equation

$$
r^{3}+3 r^{2} r_{\min }-4 r_{\min }^{3}-\frac{9}{2} Z\left(t-t_{0}\right)^{2}=0
$$

while the coordinate $\theta(t)$ follows from

$$
\beta_{0}=\sin ^{-1}\left(\frac{\cos \theta}{\cos \theta_{0}}\right)+2 \tan ^{-1}\left(\sqrt{\frac{r}{r_{\min }}-1}\right)
$$

or alternatively

$$
r \cos \theta=\cos \theta_{0}\left[\left(2 r_{\min }-r\right) \sin \beta_{0}-2 \sqrt{r_{\min }} \sqrt{r-r_{\min }} \cos \beta_{0}\right]
$$

which parallels Eq. (3.17).

\subsection{Planarity of Trajectories}

The torsion $\tau$ for the $V_{Q}$ system can be calculated from the general formula $\tau=$ - NUM/DEN given in the appendix. We thus get

$$
\begin{aligned}
\rho^{8} \times \mathrm{NUM} & =Q m\left\{\left(Q-Z \frac{\rho^{4}}{r^{3}}\right) \dot{z}+Z \frac{\rho^{2}}{r^{5}}\left[(x \dot{x}+y \dot{y})\left(r^{2}+3 z^{2}\right)-3 z \dot{z} \rho^{2}\right] z\right\} \\
\rho^{8} \times \mathrm{DEN} & =\left(Q-Z \frac{\rho^{4}}{r^{3}}\right)^{2}\left(m^{2}+\rho^{2} \dot{z}^{2}\right) \\
& +2 Z \frac{\rho^{4}}{r^{3}}\left(Q-Z \frac{\rho^{4}}{r^{3}}\right)(x \dot{x}+y \dot{y}) z \dot{z}+\left(Z \frac{\rho^{4}}{r^{3}}\right)^{2}\left(\dot{x}^{2}+\dot{y}^{2}\right) z^{2} .
\end{aligned}
$$

The condition $\tau=0$ for planarity amounts to solve

$$
\begin{aligned}
Q m\left[\left(Q-Z \frac{\rho^{4}}{r^{3}}\right) \dot{z}\right. & \left.+Z \frac{\rho^{3}}{r^{4}}(4 \dot{\rho} r-3 \dot{r} \rho) z\right] \\
& =Q m\left[\left(Q-Z \frac{\rho^{4}}{r^{3}}\right) \frac{d}{d t}(z)-z \frac{d}{d t}\left(Q-Z \frac{\rho^{4}}{r^{3}}\right)\right]=0
\end{aligned}
$$


with

$$
\begin{aligned}
& \rho r(4 \dot{\rho} r-3 \dot{r} \rho)=(x \dot{x}+y \dot{y})\left(r^{2}+3 z^{2}\right)-3 z \dot{z} \rho^{2} \\
& \rho \dot{\rho}=x \dot{x}+y \dot{y}= \pm \sqrt{\left(2 E-\dot{z}^{2}+2 Z \frac{1}{r}\right) \rho^{2}-M^{2}} .
\end{aligned}
$$

(In $(3.25)-(3.27), r$ and $\rho$ refer to spherical and cylindrical coordinates, respectively.) Equation (3.26) is satisfied for all $t$ in each of the following cases :

(i) $Q=0$. This is the case of the Kepler planetary system in which of course all trajectories are planar, namely, hyperbolas, or a parabola, or ellipses with the sun in one focus.

(ii) $z=\dot{z}=0$. The integrals of motion satisfy $|M|=\sqrt{K}$ and $\theta_{0}=\pi / 2$.

(iii) $m=0$. The motion is restricted to the plane $\varphi=\varphi_{0}$ and we have $|M|=\sqrt{Q}$.

(iv) $\rho^{4}=(Q / Z) r^{3}$. Indeed, case (iv) implies that $m=0$ (at least for bounded motions). Furthermore, the generic solution of (3.26) requires that $Q-Z\left(\rho^{4} / r^{3}\right)=c_{0} z$ with $c_{0}=$ constant, a relation which does not hold for $t$ arbitrary (except for the static case corresponding to $z=0$ and $\left.\rho^{4}=(Q / Z) r^{3}\right)$.

Consequently, in addition to the limiting case $Q=0$ (case (i)), the bounded motions are planar only if $|M|=\sqrt{K}$ (case (ii)) or $m=0$ (case (iii)).

\subsection{Periodicity and Quasi-Periodicity}

In the case of finite motions, Eq. (3.16a) allows to obtain $r$ as function of $t$ by means of a transcendental equation. The latter equation indicates that $r(t)$ is a periodic function of period

$$
T_{C}=2 \pi Z(-2 E)^{-3 / 2}
$$

a result in agreement with the fact that the parabolic coordinates $a(t)$ and $b(t)$ (with $r=(a+b) / 2)$ are also of period $T_{C}[7]$. It should be noted that the period $T_{C}$ bears exactly the same form as the one for the Coulomb-Kepler problem that corresponds to the limiting case $Q=0$. Further, from (3.16b) or (3.17) it is possible to show that $\cos [\theta(t)]$ (and 
therefore $\sin [\theta(t)])$ is a periodic function of period $T_{C}$. This result agrees with our previous work [7] due to the passage formulas $\cos \theta=(a-b) /(a+b)$ and $\sin \theta=2 \sqrt{a b} /(a+b)$. Finally, the consideration of (3.16c) leads to

$$
\varphi\left(t+k_{1} T_{C}\right)=\varphi(t)+2 k_{1} \pi \frac{m}{|M|} \quad k_{1} \in \mathbf{Z}
$$

and, therefore, the (global) motion is periodic of period

$$
T=k_{1} T_{C}
$$

if the "quantization" condition (2.34) for $|M| / m$ is fulfilled (see also Ref. [20]). As a check, in the limiting case $Q=0$, the global period $T$ of the motion is simply $T_{C}$.

In the general case $Q>0$, when the condition (2.34) is satisfied, we have the following "quantized" expressions

$$
m^{2}=Q \frac{k_{2}^{2}}{k_{1}^{2}-k_{2}^{2}} \quad \frac{r_{1} r_{2}}{r_{1}+r_{2}} \sin ^{2} \theta_{0}=\frac{1}{2} \frac{Q}{Z} \frac{k_{1}^{2}}{k_{1}^{2}-k_{2}^{2}}
$$

for the periodic bounded motions. Here again, the bounded motions for which the condition (2.34) is not satisfied are quasi-periodic motions.

The potential energy $V_{Q}$ averaged over the period $T_{C}$ (of $r$ and $\sin \theta$ ), namely,

$$
\overline{V_{Q}}=\frac{1}{T_{C}} \int_{0}^{T_{C}}\left(-Z \frac{1}{r}+\frac{1}{2} Q \frac{1}{r^{2} \sin ^{2} \theta}\right) d t
$$

cannot be calculated easily from $(3.16 a)$ and (3.16b). However, we can calculate in a straightforward way the virial of the $V_{Q}$ system. This leads to

$$
\overline{V_{Q}}=2 E+\frac{1}{2} Q \frac{1}{T_{C}} \int_{0}^{T_{C}} \frac{d t}{r^{2} \sin ^{2} \theta}=2 E+Q \frac{1}{r_{1}+r_{2}} \frac{1}{\sqrt{r_{1} r_{2}}} \frac{1}{\sin \theta_{0}}
$$

for periodic and quasi-periodic bounded motions. Therefore, the virial theorem $(\bar{T}=$ $-\bar{V} / 2=-E)$ for the three-dimensional Coulomb-Kepler problem applies to the $V_{Q}$ system only when $Q=0$.

\subsection{Particular Cases}


The case $K=M^{2}$. Equations (3.12) show that the trajectories are in the $x y$ plane when $|M|=\sqrt{K}$, a situation that corresponds to case (ii) of section 3.4. The finite trajectories are then described by

$$
\rho=2 \frac{\rho_{1} \rho_{2}}{\rho_{1}+\rho_{2}-\left(\rho_{2}-\rho_{1}\right) \sin \left[\frac{|M|}{m}\left(\varphi-\varphi_{0}\right)\right]}
$$

where $\rho_{1}$ and $\rho_{2}$ are given by (3.14) with $r \equiv \rho$. These finite trajectories are closed only if the condition (2.34) is satisfied. On the other hand, the separatrix corresponds to

$$
\rho=\rho_{\min }\left\{1+\tan ^{2}\left[\frac{1}{2} \frac{|M|}{m}\left(\varphi-\varphi_{0}\right)\right]\right\}
$$

where $\rho_{\min }$ is given by (3.21) with $r \equiv \rho$.

The case $m=0$. The trajectories are planar for $m=0$ (cf. case (iii) of section 3.4). They can be obtained in the plane $\varphi(t)=\varphi_{0}$ from (3.16a) and (3.17) and are all periodic, of period $T_{C}$.

The case $2 E K=-Z^{2}$. The finite trajectories are restricted to a sphere of radius

$$
r_{1}=r_{2} \equiv r_{0}=\frac{K}{Z}=\frac{Z}{-2 E}=\sqrt{\frac{K}{-2 E}}
$$

when $E=-(1 / 2)\left(Z^{2} / K\right)$. These trajectories (not closed in general) are described by

$$
r=r_{0} \quad z=r_{0} \cos \theta_{0} \cos \left(2 \pi \frac{t}{T_{C}}+\psi_{0}\right) \quad \varphi=\frac{m}{|M|} \tan ^{-1}\left[\frac{\tan \left(2 \pi \frac{t}{T_{C}}+\psi_{0}\right)}{\sin \theta_{0}}\right]
$$

where $\psi_{0}$ denotes a new constant. They are periodic only if the condition (2.34) for $|M| / m$ is satisfied.

\subsection{Semi-Classical Quantization}

The Bohr-Sommerfeld-Kramers quantization conditions

$$
\oint p_{r} d r=\left(n_{r}+\epsilon_{r}\right) 2 \pi \quad n_{r} \in \mathbf{N} \quad \oint p_{\theta} d \theta=\left(n_{\theta}+\epsilon_{\theta}\right) 2 \pi \quad n_{\theta} \in \mathbf{N}
$$


applied to the $V_{Q}$ system lead to

$$
2\left[S_{1}\left(r_{2}\right)-S_{1}\left(r_{1}\right)\right]=\left(n_{r}+\epsilon_{r}\right) 2 \pi \quad 2\left[S_{2}\left(\pi-\theta_{0}\right)-S_{2}\left(\theta_{0}\right)\right]=\left(n_{\theta}+\epsilon_{\theta}\right) 2 \pi .
$$

By using Eqs. (3.15), we get

$$
(-2 E)^{-1 / 2} Z-\sqrt{K}=n_{r}+\epsilon_{r} \quad-|M|+\sqrt{K}=n_{\theta}+\epsilon_{\theta}
$$

from which we recover the eigenvalue

$$
E=-\frac{1}{2} \frac{Z^{2}}{\left(|M|+n_{r}+n_{\theta}+1\right)^{2}} \quad|M|=\sqrt{m^{2}+Q} \quad m \in \mathbf{Z} \quad n_{r} \in \mathbf{N} \quad n_{\theta} \in \mathbf{N}
$$

as first derived by Hartmann [1]. Equation (3.41) implies that we must take $\epsilon_{\theta}+\epsilon_{r}=1$, a relation which reflects the occurrence of a pair of two-dimensional harmonic oscillators in the treatment of the Coulomb-Kepler problem by means of the Kustaanheimo-Stiefel transformation $[3,7]$.

\section{Closing Remarks}

We have concentrated in this paper on the classical motions for two super-integrable systems, viz, the $U_{Q}$ and $V_{Q}$ systems. The motions are always bounded for $U_{Q}$ and can be bounded or nonbounded for $V_{Q}$ just as in the limiting case $Q=0$ that corresponds to the oscillator and the Coulomb-Kepler systems in three dimensions. The bounded trajectories are confined inside simple surfaces : cylinders for $U_{Q}$ and spheres for $V_{Q}$. Both for $U_{Q}$ and $V_{Q}$, the trajectories are planar only in a few situations : (i) naturally, when $Q=0$, (ii) when

a specific relation $\left(E=K\right.$ for $U_{Q}$ and $|M|=\sqrt{K}$ for $V_{Q}$ ) exists between two constants of motion, and (iii) when the constant of motion $\ell_{3}$ vanishes. In addition, all bounded trajectories are quasi-periodic rather than periodic in general and become truly periodic if a constraint condition on $\ell_{3}$ (Eq. (2.34)), the same for $U_{Q}$ and $V_{Q}$, is satisfied. In fact, there is an infinity of periodic trajectories in the neighborhood of a given quasi-periodic trajectory since the set of the rational numbers is dense in the set of real numbers.

From a quantum mechanical point of view, the $U_{Q}$ system leads to a discrete spectrum while the spectrum for the $V_{Q}$ system comprises a discrete part, a zero point and a continuum. The Hamilton-Jacobi approach developed in sections 2 and 3, as a complement to the 
earlier study of Kibler and Winternitz [20], has allowed us to re-derive in a semi-classical way the discrete spectra for $U_{Q}$ and $V_{Q}$. For both potentials, the accidental degeneracies corresponding to fixed values of $|M|$ and $E$ are described by a unique dynamical invariance algebra, isomorphic to $s u(2)$, as shown by Quesne [2] for the $U_{Q}$ system and by Kibler and Winternitz [7] for the $V_{Q}$ system. This result is quite remarkable, especially in view of the fact that, in the limiting case $Q=0$, the dynamical invariance algebra is isomorphic to $s u(3)$ for $U_{Q=0}$ and to $s o(4)$ for $V_{Q=0}$.

As was mentioned by Kibler and Winternitz [7], the $V_{Q}$ system may for some energy levels exhibit higher degeneracies than those explained by the $S U(2)$ dynamical invariance group. These are levels characterized by the triplets $\left(m, n_{r}, n_{\theta}\right)$ and $\left(m^{\prime}, n_{r}^{\prime}, n_{\theta}^{\prime}\right)$, with $m^{\prime} \neq \pm m$, satisfying

$$
4 Q=\frac{1}{I^{2}}\left[I^{2}-\left(m+m^{\prime}\right)^{2}\right]\left[I^{2}-\left(m-m^{\prime}\right)^{2}\right] \quad I=n_{r}^{\prime}+n_{\theta}^{\prime}-n_{r}-n_{\theta} \neq 0 .
$$

A similar result holds for the $U_{Q}$ system with $I=2 n_{\rho}^{\prime}+n_{z}^{\prime}-2 n_{\rho}-n_{z}$.

Such degeneracies are "local" in that they are restricted to part of the energy spectrum of the system (cf. Ref. [24]). The quantum mechanical operators $X_{A}$ related to a "local" symmetry would not commute with the Hamiltonian but would satisfy commutation relations of the type

$$
\left[X_{A}, H\right]=\lambda\left(H-E_{A}\right)
$$

where $E_{A}$ are the energies of the levels for which the accidental degeneracy is observed. Since $I, m$, and $m^{\prime}$ are integers, Eq. (4.1) clearly imposes a constraint on the values of the coupling constant $Q$ for which additional accidental degeneracy can occur. This type of constraint is reminiscent of Eqs. (2.36) and (3.31) that also impose conditions on $Q$.

As pointed out recently by Moshinsky et al. [25], the problem of finding a group theoretical explanation of accidental degeneracy does not have an algorithmic solution. It is interesting to speculate about the relation between the periodicity condition (2.34) and the local degeneracies and we plan to return to this problem in the future. 


\section{Appendix : Torsion and Curvature for Hamiltonian Systems}

The purpose of this appendix is to derive closed formulas, in Cartesian coordinates, for the torsion and the curvature of the trajectories of a one-particle hamiltonian system. The formulas will involve solely the coordinates $\mathbf{r}(x, y, z)$ and the velocities $\dot{\mathbf{r}}(\dot{x}, \dot{y}, \dot{z})$ of the particle.

We start from the well-known formulas [26]

$$
\tau=-\frac{(\dot{\mathbf{r}} \wedge \ddot{\mathbf{r}}) . \dddot{\mathbf{r}}}{|\dot{\mathbf{r}} \wedge \ddot{\mathbf{r}}|^{2}} \quad k=\frac{|\dot{\mathbf{r}} \wedge \ddot{\mathbf{r}}|}{|\dot{\mathbf{r}}|^{3}}
$$

for the torsion $\tau$ and the curvature $k$ of a curve (in $\mathbf{R}^{3}$ ) in an arbitrary parametrization $\mathbf{r} \equiv \mathbf{r}(t)$. In the case where this curve is the trajectory of a particle (of mass $\mu$ ), we can use the Hamilton equations

$$
\dot{q}=\frac{\partial H}{\partial p} \quad \dot{p}=-\frac{\partial H}{\partial q}
$$

in order to express $\ddot{\mathbf{r}}$ and $\dddot{\mathbf{r}}$ in (A1) in terms of the Hamilton function $H \equiv H(p, q, t)$. We shall employ the variable $p$ and $q$ in the Cartesian form $\left(p_{x}=\mu \dot{x}, p_{y}=\mu \dot{y}, p_{z}=\mu \dot{z}\right)$ and $(x, y, z)$, respectively. Then, we have

$$
\ddot{x}=-\frac{1}{\mu} \frac{\partial H}{\partial x} \quad \ddot{y}=-\frac{1}{\mu} \frac{\partial H}{\partial y} \quad \ddot{z}=-\frac{1}{\mu} \frac{\partial H}{\partial z}
$$

and

$$
\dddot{x}=-\frac{1}{\mu}\left(\frac{\partial^{2} H}{\partial x^{2}} \dot{x}+\frac{\partial^{2} H}{\partial y \partial x} \dot{y}+\frac{\partial^{2} H}{\partial z \partial x} \dot{z}-\frac{\partial^{2} H}{\partial p_{x} \partial x} \frac{\partial H}{\partial x}-\frac{\partial^{2} H}{\partial p_{y} \partial x} \frac{\partial H}{\partial y}-\frac{\partial^{2} H}{\partial p_{z} \partial x} \frac{\partial H}{\partial z}+\frac{\partial^{2} H}{\partial t \partial x}\right)
$$

with similar expressions for $\dddot{y}$ and $\dddot{z}$. As a result, the quantities $\tau$ and $k$ can be developed in terms of $(\dot{x}, \dot{y}, \dot{z})$ and of the derivatives of $H$ which turn out to be functions of $(x, y, z)$, $(\dot{x}, \dot{y}, \dot{z})$ and $t$. This leads to formulas, not reported here, which are intricate but very easy to handle with a symbolic computer language like Reduce or Macsyma.

We now restrict ourselves to the case of conservative systems for which

$$
H=\frac{1}{2 \mu}\left(p_{x}^{2}+p_{y}^{2}+p_{z}^{2}\right)+V(x, y, z)
$$


Then, the expressions for

$$
\tau=-\frac{\mathrm{NUM}}{\mathrm{DEN}} \quad k=\frac{\sqrt{\mathrm{DEN}}}{\operatorname{den}} \quad \operatorname{den}=\left(\dot{x}^{2}+\dot{y}^{2}+\dot{z}^{2}\right)^{3 / 2}
$$

can be given a simple form. Indeed, we get

$$
\begin{aligned}
\mu^{2} \times \mathrm{NUM} & =\left(\dot{y} \frac{\partial V}{\partial z}-\dot{z} \frac{\partial V}{\partial y}\right)\left(\frac{\partial^{2} V}{\partial x^{2}} \dot{x}+\frac{\partial^{2} V}{\partial y \partial x} \dot{y}+\frac{\partial^{2} V}{\partial z \partial x} \dot{z}\right) \\
& +\left(\dot{z} \frac{\partial V}{\partial x}-\dot{x} \frac{\partial V}{\partial z}\right)\left(\frac{\partial^{2} V}{\partial x \partial y} \dot{x}+\frac{\partial^{2} V}{\partial y^{2}} \dot{y}+\frac{\partial^{2} V}{\partial z \partial y} \dot{z}\right) \\
& +\left(\dot{x} \frac{\partial V}{\partial y}-\dot{y} \frac{\partial V}{\partial x}\right)\left(\frac{\partial^{2} V}{\partial x \partial z} \dot{x}+\frac{\partial^{2} V}{\partial y \partial z} \dot{y}+\frac{\partial^{2} V}{\partial z^{2}} \dot{z}\right) \\
\mu^{2} \times \mathrm{DEN} & =\left(\dot{y} \frac{\partial V}{\partial z}-\dot{z} \frac{\partial V}{\partial y}\right)^{2}+\left(\dot{z} \frac{\partial V}{\partial x}-\dot{x} \frac{\partial V}{\partial z}\right)^{2}+\left(\dot{x} \frac{\partial V}{\partial y}-\dot{y} \frac{\partial V}{\partial x}\right)^{2}
\end{aligned}
$$

or in compact form

$$
\mathrm{NUM}=\frac{1}{\mu^{2}}(\dot{\mathbf{r}} \wedge \nabla V) \cdot\left(\left(\dot{\mathbf{r}} \cdot \nabla \frac{\partial V}{\partial x}\right) \mathbf{i}+\left(\dot{\mathbf{r}} \cdot \nabla \frac{\partial V}{\partial y}\right) \mathbf{j}+\left(\dot{\mathbf{r}} \cdot \nabla \frac{\partial V}{\partial z}\right) \mathbf{k}\right) \quad \text { DEN }=\frac{1}{\mu^{2}}|\dot{\mathbf{r}} \wedge \nabla V|^{2}
$$

so that $\tau$ and $k$ are given by closed form expressions involving only $(x, y, z)$ and $(\dot{x}, \dot{y}, \dot{z})$. Equations (A6) and (A7) are applied to $V=U_{Q}$ and $V=V_{Q}$ in the main body of this paper.

\section{Acknowledgments}

This work has been achieved in the framework of the France-Québec exchange programme (project no 200220 89). The kind hospitality extended to one of the authors (M. K.) at the Centre de Recherches Mathématiques de l'Université de Montréal on the occasion of several stays during the work on this project is gratefully acknowledged. The research of one of the authors (P. W.) is partially supported by research grants from NSERC of Canada and FCAR of Québec. Two of the authors (M. K. and P. W.) are indebted to Dr. N. W. Evans from the Queen Mary College (London) for sending them preprints on his work and to Professor Ya. A. Smorodinsky for an interesting discussion. 


\section{Bibliography}

[1] H. Hartmann, in : Sitzungsberichte der wissenschaftlichen Gesellschaft der J.-W. Goethe Universität, Frankfurt am Main 10, 107 (1972) ; ibid. Theor. Chim. Acta 24, 201 (1972) ; H. Hartmann, R. Schuck, and J. Radtke, Theor. Chim. Acta 42, 1 (1976) ; D. Schuch, Master Thesis, J.-W. Goethe Universität, Frankfurt am Main (1978) ; H. Hartmann and D. Schuch, Int. J. Quantum Chem. 18, 125 (1980).

[2] C. Quesne, J. Phys. A : Math. Gen. 21, 3093 (1988).

[3] M. Kibler and T. Négadi, Int. J. Quantum Chem. 26, 405 (1984) ; ibid. Croat. Chem. Acta 57, 1509 (1984).

[4] M. V. Carpio and A. Inomata, in : Path Integrals from meV to MeV, Eds. M. C. Gutzwiller, A. Inomata, J. R. Klauder, and L. Streit (Singapore : World Scientific, 1986).

[5] I. Sökmen, Phys. Lett. 115A, 249 (1986).

[6] C. C. Gerry, Phys. Lett. 118A, 445 (1986).

[7] M. Kibler and P. Winternitz, J. Phys. A : Math. Gen. 20, 4097 (1987).

[8] L. Chetouani, L. Guechi, and T. F. Hammann, Phys. Lett. 125A, 277 (1987).

[9] I. V. Lutsenko, G. S. Pogosyan, A. N. Sissakyan, and V. M. Ter-Antonyan, Teor. i Mat. Fiz. 83, 419 (1990).

[10] M. V. Carpio-Bernido and C. C. Bernido, Phys. Lett. 134A, 395 (1989) ; ibid. Phys. Lett. 137A, 1 (1989) ; M. V. Carpio-Bernido, J. Phys. A : Math. Gen. 24, 3013 (1991) ; ibid. J. Math. Phys. 32, 1799 (1991).

[11] J. M. Cai and A. Inomata, Phys. Lett. 141A, 315 (1989).

[12] A. N. Sissakian et al., preprint P2-89-814, JINR, Dubna (1989). 
[13] H. Boschi Filho and A. N. Vaidya, Phys. Lett. 145A, 69 (1990) ; ibid. Phys. Lett. 149A, 336 (1990) ; H. Boschi-Filho, M. de Souza, and A. N. Vaidya, J. Phys. A : Math. Gen. 24, 4981 (1991).

[14] O. F. Gal'bert, Ya. I. Granovskii, and A.S. Zhedanov, Phys. Lett. 153A, 177 (1991).

[15] A. Guha and S. Mukherjee, J. Math. Phys. 28, 840 (1987).

[16] M. Kibler and T. Négadi, Phys. Lett. 124A, 42 (1987) ; ibid. in : Proc. 17th Int. Colloq. on Group Theoretical Methods in Physics, Eds. Y. Saint-Aubin and L. Vinet (Singapore : World Scientific, 1989).

[17] I. Sökmen, Phys. Lett. 132A, 65 (1988).

[18] L. Chetouani, L. Guechi, and T. F. Hammann, J. Math. Phys. 30, 655 (1989).

[19] H. D. Doebner and E. Papp, Phys. Lett. 144A, 423 (1990).

[20] M. Kibler and P. Winternitz, Phys. Lett. 147A, 338 (1990).

[21] P. Winternitz, Ya. A. Smorodinskiı̌, M. Uhlír, and I. Friš, Yad. Fiz. 4, 625 (1966) [Sov. J. Nucl. Phys. 4, 444 (1967)] ; see also : I. Friš, V. Mandrosov, Ya. A. Smorodinsky, M. Uhlír, and P. Winternitz, Phys. Lett. 16, 354 (1965).

[22] A. A. Makarov, J. A. Smorodinsky, Kh. Valiev, and P. Winternitz, Nuovo Cimento A 52, 1061 (1967).

[23] N. W. Evans, Phys. Lett. 147A, 483 (1990) ; ibid. Phys. Rev. A 41, 5666 (1990) ; ibid. J. Math. Phys. 31, 600 (1990).

[24] A. Joseph, Int. J. Quantum Chem. 1, 535 (1967) ; see also : C. A. Coulson and A. Joseph, Int. J. Quantum Chem. 1, 337 (1967).

[25] M. Moshinsky, C. Quesne, and G. Loyola, Ann. Physics 198, 103 (1990).

[26] M. P. do Carmo, Differential Geometry of Curves and Surfaces (New Jersey : Prentice-Hall, 1976) 